\title{
The Impacts of Blink on Graphical Information Processing
}

\author{
Gia Kim \\ School of Business (IT) \\ James Cook University \\ Cairns, Australia \\ Gia.Kim@juc.edu.au
}

\author{
Siu Man Lui \\ School of Business (IT) \\ James Cook University \\ Cairns, Australia \\ Carrie.Lui@jcu.edu.au
}

doi: 10.4156/ijipm.vol1.issue2.15

\begin{abstract}
Dynamic graphs presenting huge amount of time-series data have become more and more commonly used these days. Although previous research revealed that blinking component can attract human attention, hence facilitates visual, the effect of blink have not been clearly evident in a dynamic graph reading context. This study examines the effects of blink on the user's affective experience and usability of using a blinking line graph. Additionally, this study describes an empirical experiment setup for investigating the characteristics of task types as a moderator to the relationship between blink and the user's experience. This research aims to theoretically contribute graph comprehension domain by investigating the effects of blink on the graph comprehension process by providing a literature review about the influence of blink and task types on graph reading effectiveness and propose a quantitative experiment and to propose possible hypotheses.
\end{abstract}

Keywords: Graph, Attention; Affect; Response time; Accuracy

\section{Introduction}

This study investigates the impacts of blink on users' affective experience and task performance when using blinking line graphs. Blink refers to a type of visual animation element that stays at the identical position with alternating a visual figure 'on and off' [1,2]. Animation is defined as a series of graphics that change over time. That is why animation is also called motion graphics. Motion is found to have a stronger power to grab human attention than any other visual elements, such as color and orientation [3,4]. Although blink does not display orientation or movement, its feature of shifting between on and off generates influential attraction for human visual attention. It is believed that such an 'on and off' feature of blink would increase viewers' arousal states [5].

Both researchers and practitioners have been concerned how blink attracts human attention. In practice, multimedia designers have used blink to attract attention [6-8]. Moreover, blink is used as an alarm in information visualization to attract the user's attention for emergency information or critical events [9]. Blink is also used for visual search in dynamic displays and websites. Scholars have discovered that dynamic stimuli, such as the speed of animation, the direction of motion, and audio, catch attention. These dynamic stimuli facilitate information processing and searching $[4,5]$. Animation, as a moving object, is believed to capture human peripheral vision regardless of whether the animated item is a target or non-target for a task. Prior researchers of dynamic displays argued that time-control coded displays, such as blink coding and animation, but especially blink coding, are effective attention-getting tools [1] . The relationship of blink and human attention continues to be discussed in academic and business circles.

Blink is widely used for web advertising banners to attract the potential online buyer's attention and to increase the probability to click through the advertisement. Blinking photos or descriptions of the selling product are common in online shopping websites. It is believed that blinking items may help the shopper's search for their target products easily. Sometimes, blink is used to highlight special products on the webpage. 
However, many usability researchers have consistently been critical of the use of blink; because they argue that it significantly creates a negative affective experience $[1,2,10]$. Nielsen [10] found that $87 \%$ of online users simply hate the use of blinking advertisements on websites. Lou Montulli, an inventor of the blink HTML tag, mentioned that blink is extremely user-unfriendly, and it is the worst thing he has ever done for the Internet. It is believed that the blinking message reduces readability [1]. Furthermore, blink increases human sight's tension. Looking at persistently running animation like blink is not only tiring but also tremendously dangerous under certain conditions, if designers do not understand the proper use of blink. It is reported on 16th December, 1997 that approximately 700 viewers, mostly children, displayed seizure symptoms when watching the Japanese TV animation 'Pocket Monsters' [11]. The scene showed a low luminance $12 \mathrm{~Hz}$ red and blue color flicker stimulus across an entire wide screen.

Although blink is commonly used in the real world, it is not always useful for the user. Nielsen [10] argued that blink is only useful when the user is trying to distinguish an item from a large amount of items on the screen. Hong, Thong, and Tam [12,13] also discovered that a blinking item can be distinguished more easily, and the effect is stronger especially among items located closely to one another. In order to use blink in an effective way, it is important to understand the feature of blink and the characteristics of the tasks that blink may facilitate.

This study aims to investigate the effects of blink on the user's affect and usability when using a 2D line graph. In addition, the moderation effects of task types to the impacts of blink will also be investigated.

In the empirical study, two graphs will be used for understanding the effects of blink. One includes one blinking line and two other static lines, and the other includes all three static lines, that is, a non-blinking condition. The experiment examines the user's affective response and usability in terms of response time, accuracy, and satisfaction. Changes, a feature of animation, grab the attention of human eyes. Visual attention affects the user's performance with the item they look at. The user's response time has been used in Information System research as a traditional measure for the efficiency of the user's performance [14]. It is assumed that the user can complete the task in a shorter duration when the graphs are designed to present the information properly.

Another focus of this study is to investigate the characteristics of task types that may possibly influence the user's affective response and usability with a blinking graph. The complexity of task types influences the user's affective experience and the level of workload. Elementary tasks refer to simple tasks that can be completed through a single-step of mental workload; for example, grasping the big-picture of the trend and reading the details of data values $[15,16]$. Advanced tasks refer to the tasks containing higher complexity that can be completed through multiple steps. They also require a more complicated mental workload [1517]. For example, complex tasks involve reading and calculating values, and then comparing them. Tasks of gathering visual information by eyes and comparing their sizes are comparatively easier. Such a high level of information load demands concentrated attention over the information processing.

\section{Theoretical Background}

Blink is designed to attract human attention by using a human physiological condition of automatically looking at moving objects. It helps the viewer to search important information as quickly as possible. Thus, the feature is ideal for alarm that warns the user to be alert for any danger. Alarm must attract the user's attention and facilitate the use to finish tasks as quickly as possible under a time-critical condition.

Information visualization for complex and large amount of time-series data has been researched in Information Technology [18-20]. Line graphs, bar charts, and their combination, candle charts, are commonly used in the financial industry to present real-time and time-series changes. Line graphs in particular, are universally used, as one of the basic types of graph documentation, in other business areas as well as in academics.

Graphs have been studied for a long time for the purpose of teaching young students mathematics and science [21, 22]. Researchers have been interested in how the viewer 
understands graphs and obtains information from them. Prior research proposed that different spatial techniques are used by the graph-reader to process and to understand the information presented on the graph [23-25]. Trickett and Trafton [24, 25] found that the graph-reader uses spatial transformation to understand the spatial characteristics of the graph. Hollands and Spence [23] found that judging part-to-whole relationships with bar charts took longer than with pie charts or divided bar charts. These bar charts (simple bar, divided bar, and reference bar) and pie charts have been discussed with the aspect of the viewer's size judgment whilst line graphs have a limitation to display sizes [21, 23, 25, 26]. Hereupon, others have approached line graphs with a focus on the characteristics of them by comparing simple lines and a $3 \mathrm{D}$ version of line graphs [17,27]. Kumar and Benbasat [17] argued that the user makes inferences better with 3D line graphs with more than two variables than 2D line graphs. Most of these researches have used static graphs, and thus the validity of these findings for blinking graphs is uncertain. Since the Internet was universalized, dynamic real-time graphs have been progressively more common in every day life. There is a need for more research attention on blinking graphs to understand how they can be used more effectively.

Researchers on dynamic displays have examined the impacts of blink on the display comprehension. Blink has been investigated in visual search studies as an aspect of a visual factor. Web researchers have examined the effects of blink on web usability [10]. They have tested blink with words or sentences of texts online and blink with images. The results suggested that using blink on the website reduces readability of the text. However, readability of graphs using blink is different from the one with texts and images because graph comprehension is different from information processing of texts or images.

A graph can contain a vast amount of information. However, not all of the information is always important to the user. What information is valuable to the user depends on tasks and situations. Under a time-critical situation, it is important for the graph-reader to be able to extract useful information from the graph as quickly and accurately as possible. At the same time, it is also important to enable the graph-reader to ignore the irrelevant information. Therefore, this study investigates whether blink can play the role of a facilitator or a distracter in time-critical contexts.

The empirical experiment of this study is designed based on a literature review of the following three theoretical domains: 1) graph comprehension, 2) attention capture, and 3) information processing.

\subsection{Graph Comprehension}

Graph comprehension theories explain how the graph-reader understands graphs. The elementary processes of the viewer's extracting information from graphs are anchoring, scanning, projection, superimposition, and detection operators [26, 28]. According to Pinker's graph comprehension framework, bar charts, pie charts, and line graphs have their own graph schemas. The viewer first tries to understand the graph schema and uses it as a guide to obtain information from the graph. Trickett and Trafton [24, 25] proposed the concept of spatial transformations. They found that the viewer uses spatial transformation to read information from graphs. Human eyes first locate the area of visual cues. To extract the related information, the viewer draws invisible guidelines in their mind. They try to match the visual cue and the value using these guidelines.

Most graph comprehension researchers believe that generally the following five factors, including display characteristics, data complexity, task types, background knowledge, and the viewer's graph knowledge, influence graph reading performance [25]. This study is focused on display characteristics and task types. Previous studies have suggested a range of tasks from elementary to advanced levels [21]. Elementary tasks ask to extract information from the data in the graph by reading a single specific point. For example, 'What is the value of the pie-slice A?'. Intermediate tasks ask to find relationships in the data that needs attention to more than a single specific point. For example, 'Is A + B equal to C + D?'. Last, overall tasks ask to move beyond the data. The questions often ask to capture overall specific values of the graph. For example, 'For the period of 15th June to 30th June, what was the trend for the value of Stock X?'. 
Because of the level of complexity of overall tasks, they have often been treated as advanced level tasks. Prior research has found that graph design has different impacts on elementary and advanced tasks. The result of the user's response may vary depending on their methods of answering, like typing values in the text field or clicking or dragging values on the graph itself.

Simkin and Hastie [26] categorized three tasks with graphs: discrimination, comparison, and proportion judgment. In this study, first, the user is asked to extract the highest value and the lowest value of a line for an ability of discrimination. The second type of task is to compare values of two lines for particular periods. Last, the user is asked to estimate the size of ranges between one line and another.

\subsection{Visual Attention Capture}

Another theoretical foundation for designing of a blinking graph is attention capture. A salient visual feature attracts attention on the computer screen. Attention and psychology research shows distinctive aspects of that a sight captures human attention. For example, visually unique scenes and unexpected features hold attention. Brockmole and Boot [29, 30] discovered that while the colors of all objects changed, unexpected novel color and single unique color captured more attention. This feature is valid even thought the distinctive items are task-irrelevant stimuli.

According to a visual search study, users perform faster when they obtain information of items with a salient feature [13]. Many prior investigators on visual search tasks have discovered that a dynamic target among static distracters holds human attention almost automatically. In other words, the user can easily distinguish the blinking item from other nonblinking items without effort. Interestingly, it is also found that a static item among dynamic distracters is distinguished as easily as a blinking item among other static items [5]. This distinctive feature is a key factor of attention capture.

Unique, distinctive, and special features have already been used as the means of capturing attention in visual communication. A vivid color among calm colors, different shapes from surrounding, and an opposite orientation to the one of background are examples of salient features for attention capture.

Motion is another important factor for attention capture. The user's interaction with the moving item will be more efficient than when dealing with a set of motionless objects. However, many believe that the difference between the salient feature of motion and the other stimuli, including color and orientation, is that motion is automatic capture of attention [4]. Other motionless stimuli tend not to automatically capture human attention although they hold the salient feature.

In the graph reading context, an attention mechanism is explained that 'attention-capturing' graph schemas provide the viewer to search relevant information [4]. When the target item appears on the graph, the attention-capturing schema allows the target to be selected from among the distracting items.

Another difference of the blinking graph reading study to prior visual search studies, as an aspect of salient feature, is that a blinking line is not an actual target for search but it is a target line matching with relevant information the user is looking for. The user's graph-reading examines not only the salient feature of blinking lines but also another step of information processing, which is reading information from the blinking line or non-blinking lines. Tasks in this study are sometimes asked using a blinking line and a non-blinking line together to sort out an advanced level of information extraction. This characteristic is the main difference from dynamic visual search studies, and thus, this study may further expand existing attention capture theories.

Lastly, the meaning of a blinking line as a salient feature in this study relies on density of lines. Hong, Thong, and Tam [13] found that blinking items attract considerable attention under higher local density rather than low local density. This means the user quickly searches a blinking item among items located closely to one another than the ones located far away from one another. The salient feature in the later condition is less noticeable, and thus, it attracts less attention. Similarly, density of lines could be explained as the number of intersections from one 
line to another. From everyday experience, daily or monthly line charts of stock market data present a significant amount of intersections between multiple lines. Often, the more lines are in a graph, for instance, a share price line, a 3-day moving average line, a 5-day moving average line, a 10-day moving average line, and so on, the more chances there are to intersect between one line and another.

\subsection{Affect in Information Processing}

Research in cognitive science, neuroscience, and psychology revealed that human beings employ cognitive and affective channels to process information [31,32]. Cognitive processes and affective processes interact with each other during the human information process. Norman, Ortony and Russell [33] argued that visual stimuli sensed by human sensory systems pass through three levels of processing: visceral, behavioral, and reflective levels. The visceral level makes quick judgments like good or bad and sends signals to the muscles. Affective processing begins expressing relaxation or tension on the muscles immediately. This level alerts the rest of the brain. Next, the behavioral level makes the body act unconsciously, and lastly the reflective level thinks consciously based on the past experience and memories. These layers interact with one another until human muscles respond.

Researchers have also investigated affect in relation to human information processing, visual perception, and cognitive processing [26, 28]. Sun and Zhang [34] studied the role of affect in information technology acceptance process. They systemized the framework of the impact of the user's affective reaction towards using information technology. Their framework suggested that affective reaction and cognitive reaction interact with each other through the process.

Previous research on information processing shows that the impact of negative affect on the brain process is as important as positive affect [35]. Negative affect like tension and stress is essential to escape from dangerous situations in real life. The visceral level of judgments of whether the information is safe or dangerous strongly interacts with negative affect. When people are anxious, they tend to narrow their information processes, not to care about any other issues but just concentrating on only the relevant issue in front of them. This actually facilitates urgent problem solving, which is believed as an advantage of generating negative affect intentionally or accidently. Reading numbers and extracting specific values from the graph are to process detail information, which could be benefit from the concentrated and focused processing driven by negative affect.

On the other hand, positive affect widens brain process [35]. Positive affect helps the user think in terms of unrestricted concept and creative ideas. Using this principle, observing big pictures of the graph without extraction of specific data values could benefit from positive affect. Thus, human brains function in a relaxed and more creative way while looking at the big picture.

The influence of affect on information processing even starts one step before the actual event of brain process on the timeline [36]. Reeves and Nass [36] discovered that if the user was in a state of arousal for some reason before watching arousal news, they respond more sensitively to the news story from media. For example, when the person had a fight with friend and then watched an arousing scene, his or her level of arousal would be higher than someone who did something calming before watching it. Furthermore, their research argued that positive arousing segments could possibly intensify one's response to a subsequent negative arousing scene.

The connection between affect and visual information process guides the design tasks of reading a blinking graph.

\section{Methodology}

This study examines the effects of blink on the user's affect and usability when using a blinking line graph. In addition, the moderation effect of task types to the impacts of blink will also be investigated. The user's responses to a graph that consists of a blinking line and two static lines and another graph that consists of all non-blinking lines will be empirically tested. 
Also, tasks for obtaining big-pictures and tasks for extracting details will be used for investing the moderation effect.

The participants will be randomly divided into two groups. One group of users is asked a range of tasks from reading values of a point of the blinking line or non-blinking line to calculation of the difference between the highest value and the lowest value. This means the user is required to work with a single blinking line, a single non-blinking line, and both a blinking and a non-blinking line for a task. The other group of users will conduct the same tasks with all static lines.

A blinking line in this experiment is presented using $1 \mathrm{px}$ line thickness for one second, as a default, and altered using 2px line thickness for another one second. The whole line is blinking and it remains at the same speed until the end of the experiment.

Two dependent variables will be used to measure the efficiency of the user performance on usability: response time and accuracy $[14,37]$. In this experiment, if the graph is easy to use, the user will complete their tasks quickly and accurately. Efficiency of user performance refers to the state without hesitation while conducting tasks. If there are distractions like difficulty or ambiguity, the completing time is affected directly. Degree of accuracy shows how the user is engaging with the tasks.

Two other dependent variables used in the study are affect and satisfaction. Satisfaction is the user's final evaluation. Likability, that is, whether the user likes the blink or not, and attitudes towards the interface of the graph are measured for satisfaction. Overall satisfaction asks if the user finds the graph with tasks enjoyable to use, exciting, pleasant, interesting, etc. Affect is used to measure the user's attentiveness, that is, how much they are engaging with the graph. Also affect checks how much the blink of the graph evokes the user's negative or positive state during the task.

\section{Hypotheses Development}

\subsection{Big-Picture Tasks: Blink vs. No Blink}

The characteristic of a big-picture task is that it should not be complicated. For example, a task for a big picture is to identify the trend of the graph whether the line is increasing or decreasing. Trends are technically recognized by comparing the sequence of high and low values. When each subsequent high is higher than the previous high, while each subs equent low is also higher than the preview low, this graph is called uptrend. Downtrend is defined in the same manner; when each high is lower than the previous one and each low is lower than the previous one.

Based on brain processing theories, human brains do not use the technical method above to obtain all the high and low values and compare the previous ones to the next. Human brains simply observe the big picture of the graph trend. The viewer is able to get one big picture at one glance. Blink for this simple task is rather distracting because blink grabs attention and thus, the viewer is likely to look at lines longer than enough.

H1a. Response time for big-picture tasks will be shorter when there is no blink.

The characteristic of a big picture is also to reduce the possibility to answer incorrectly because there are only two choices (e.g. increase or decrease). Even capturing the range from the highest value to the lowest value of two lines and comparing which one is larger is the advanced level of task obtaining a big picture of the graph. However, it is still comparatively simple because there are also only two choices (e.g. Line A or Line B). The flash feature of blink may cause any mistakes to read wrong labels.

H1b. Response for big-picture tasks will be more accurate when there is no blink.

Big-picture tasks do not require focus but just observing a general idea of the graph. Blink for a big-picture task is unlikely to provide a positive influence. Blink may annoy the user 
gathering general information, rather than specific information. All static lines make the user relaxed and thus, may allow them perform better in this case. As a result, big-picture tasks with all static lines are likely to satisfy the user.

H1c. The user will experience more negative affect for big-picture tasks when there is blink. H1d. The user will be more satisfied at big-picture tasks when there is no blink.

\subsection{Detailed Tasks: Blink vs. No Blink}

In contrast, detailed tasks require generally more time to complete and demand focus over the task. The tasks ask to identify a specific point of the line and to read the value of the point on $\mathrm{Y}$-axis then to type the number into the text field. Although it is an easy reading-value task, it requires the process of graph comprehension [28]. The user should be more attentive than when they conduct observing-trend tasks.

At an advanced level, the user is asked to indentify all the periods of where the values of a line are higher than the ones of the other one. This task needs to be worked with two lines. Blink may create a higher level of contrast between two lines [12]. Higher contrast provides easier distinction.

H2a. Response time for detailed tasks will be shorter when there is blink.

$\mathrm{H} 2 \mathrm{~b}$. Response for detailed tasks will be more accurate when there is blink.

Blink is refreshing human sight by providing tension into the visual nerve $[31,32,38]$. Sight tension and nervousness make the user remain in focus on complex jobs. Hence, blink may function better with detailed tasks. Although blink generates a slight negative affect due to its characteristic, overall satisfaction of detailed tasks with a blink line is likely to be higher than when working with a non-blinking graph.

$\mathrm{H} 2 \mathrm{c}$. The user will experience more negative affect for detailed tasks when there is blink.

$\mathrm{H} 2 \mathrm{~d}$. The user will be more satisfied at detailed tasks when there is blink.

\section{Conclusion}

Overall, this study aims to contribute in three areas. First, the comparis on between the blinking line and the non-blinking line, as an aspect of graph comprehension, has been received little attention in the literature. Second, it examines objective usability of response time and accuracy as well as a subjective affective state of using a blinking line graph. Both significantly influence user satisfaction of information displays. The relationship between affect and easy-ofuse and usefulness will be discussed in further research. Third, this research investigates the moderating effects of task types with the information presentation of a blinking visual feature. This aspect has rarely received attention by researchers, particularly the connection between the salient feature of blink and the complexity of task types.

\section{References}

[1] A. Morse, "Some principles for the effective display of data," ACM SIGGRAPH Computer Graphics, vol. 13, 1979, pp. 94-101, doi:ACM O-89791-004-4/79/0800-094.

[2] S. L. Smith and N. C. Goodwin, "Blink coding for information display," Human Factor, vol. 13. 1971, pp. 283-290.

[3] J. H. R. Maunsell and S. Treue, "Feature-based attention in visual cortex," Trends in Neurosciences, vol. 29, June. 2006, pp. 317-322, doi:10.1016/j.tins.2006.04.001.

[4] M. Girelli and S. J. Luck, "Are the same attentional mechanisms used to detect visual search targets defined by color, orientation, and motion?," Journal of Cognitive Neuroscience, vol. 9, 1997, pp. 238-253. 
[5] Y. Pinto, C. N. L. Olivers, and J. Theeuwes, "When is search for a static target among dynamic distractors efficient?," Journal of Experimental Psychology: Human Perception and Performance, vol. 32, 2006, pp. 59-72, doi:10.1037/0096-1523.32.1.59.

[6] J. Hartmann, A. Sutcliffe, and A. D. Angeli. "Investigating attractiveness in Web User Interfaces," Proc. SIGCHI Conf. Human Factors in Computing Systems: Empirical Studies of Web Interaction (CHI 07), ACM Press, Apr. 2007, pp. 387-396, doi:ACM 978-1-59593-593-9/07/0004.

[7] I. Nakarada-Kordic and B. Lobb. "Effect of perceived attractiveness of web interface design on visual search of web sites," Proc. 6th ACM SIGCHI New Zealand Chapter's Inter. Conf. Computer-Human Interaction: Making CHI Natural (CHINZ 05), ACM Press, July. 2005, pp. 2527, doi:ACM 1-59593-036-1/04/10.

[8] W. Lee and I. Benbasat, "Designing an electronic commerce interface: Attention and product memory as elicited by web design," Electronic Commerce Research and Applications, vol. 2, Apr. 2003, pp. 240-253, doi:10.1016/S1567-4223(03)00026-7.

[9] N. Gershon and W. Page, "What storytelling can do for Information Visualization," Communications of the ACM, vol. 44, Aug. 2001, pp. 31-37.

[10] J. Nielsen and H. Loranger, Prioritizing Web Usability. C. Peri, Ed. Berkeley, CA: New Riders, 2006.

[11]T. Takahashi and Y. Tsukahara, "Pocket Monster incident and low luminance visual stimuli: Special reference to deep red flicker stimulation," Acfa Faediafrica Japonica, vol. 40, May. 1998, pp. 631-637.

[12] W. Hong, J. Y. L. Thong, and K. Y. Tam, "How do web users respond to non-banner-Ads animation? The Effects of Task Type and User Experience," Journal of the American Society for Information Science and Technology, vol. 58, June. 2007, pp. 1467-1482, doi:10.1002/asi.20624.

[13] W. Hong, J. Y. L. Thong, and K. Y. Tam, "Does animation attract online users' attention?: The effects of flash on information search performance and perceptions," Information Systems Research, vol. 15, Mar. 2004, pp. 60-86, doi:10.1287/isre.1040.0017.

[14]K. Hornbæk, "Current practice in measuring usability: Challenges to usability studies and research," International Journal of Human-Computer Studies, vol. 64, Feb. 2006, pp. 79-102, doi:10.1016/j.ijhcs.2005.06.002.

[15]I. Zigurs and B. K. Buckland, "A theory of Task/Technology Fit and Group Support Systems effectiveness," MIS Quarterly, vol. 22, Sep. 1998, pp. 313-334.

[16]D. J. Campbell, "Task complexity: A review and analysis," The Academy of Management Review, vol. 13, Jan. 1988, pp. 40-52.

[17] N. Kumar and I. Benbasat, "The effect of relationship encoding, task type, and complexity on information representation: An empirical evaluation of 2D and 3D line graphs," MIS Quarterly, vol. 28, June. 2004, pp. 255-281.

[18] S. P. Callahan, et al. "VisTrails: Visualization meets data management," Proc. ACM SIGMOD Inter. Conf. Management of Data (SIGMOD 06), ACM Press, Jun. 2006, pp. 745-747, doi:ACM $1595932569 / 06 / 0006$.

[19] V. Mendoza and D. G. Novick. "Usability over time," Proc. 23rd Annual Inter. Conf. Design of Communication: Documenting \& Designing for Pervasive Information (SIGDOC 05), ACM Press, Sept. 2005, pp. 151-158, doi:ACM 1-59593-175-9/05/0009.

[20]E. Karapanos, M. Hassenzahl, and J-B. Martens. "User experience over time," Proc. Works in Progress. Human Factors in Computing Systems (CHI 08), ACM Press, Apr. 2008, pp. 3561-3566, doi:ACM 978-1-60558-012-8/08/04.

[21] S. N. Friel, F. R. Curcio, and G. W. Bright, "Making sense of graphs: Critical factors influencing comprehension and instructional implications," Journal for Research in Mathematics Education, vol. 32, Mar. 2001, pp. 124-158.

[22] M. Hegarty, "Dynamic visualizations and learning: getting to the difficult questions," Learning and Instruction, vol. 14, 2004, pp. 343-351.

[23] J. G. Hollands and I. Spence, "Judging proportion with graphs: The summation model," Applied Cognitive Psychology, vol. 12, 1998, pp. 173-190, doi:CCC 0888-4080/98/020173-18.

[24] S. B. Trickett and J. G. Trafton, "Spatial transformations in graph comprehension," in Diagrams, A. Blackwell, Ed. Berlin Heidelberg: Springer-Verlag, 2004, pp. 372-375. 
[25] S. B. Trickett and J. G. Trafton, "Toward a comprehensive model of graph comprehension: Making the case for spatial cognition," in Diagrams, D. Barker-Plummer, Ed. Berlin Heidelberg: Springer-Verlag, 2006, pp. 286-300.

[26] D. Simkin and R. Hastie, "An information-processing analysis of graph perception," Journal of the American Statistical Association, vol. 82, June. 1987, pp. 454-465.

[27]P. Shah and P. A. Carpenter, "Conceptual limitations in comprehending line graphs," Journal of Experimental Psychology: General, vol. 124, 1995, pp. 43-61.

[28] S. Pinker, "A theory of graph comprehension," in Artificial Intelligence and the Future of Testing, R. Feedle, Ed. 1990. pp. 73-126.

[29] G. L. West, A. A. K. Anderson, and J. Pratt, "Motivationally significant stimuli show visual prior entry: Evidence for attentional capture." Journal of Experimental Psychology: Human Perception and Performance, vol. 35, 2009, pp. 1032-1042, doi:10.1037/a0014493.

[30] J. R. Brockmole and W. R. Boot, "Should I stay or should I go? Attentional disengagement from visually unique and unexpected items at fixation," Journal of Experimental Psychology: Human Perception and Performance, vol. 35, 2009, pp. 808-815, doi:10.1037/a0013707.

[31] C. M. Bloomer, Principles of Visual Perception. London: The Herbert Press, 1990.

[32] I. E. Gordon, Theories of Visual Perception, 3rd ed. New York: Psychology Press, 2004.

[33]D. A. Norman, A. Ortony, and D. M. Russell, "Affect and machine design: Lessons for the development of autonomous machines," IBM Systems Journal, vol. 42, 2003, pp. 38-44.

[34]H. Sun and P. Zhang, "The role of affect in Information Systems research: A critical survey and a research model," in HCI in MIS (I): Foundations, P. Zhang and D. Galletta, Eds. Sharpe Publisher, 2005; pp. 333-333.

[35]D. A. Norman, Emotional design: Why We Love (or Hate) Everyday Things. New York: Basic Books, 2004, pp. 21-29.

[36] B. Reeves and C. Nass, The Media Equation: How People Treat Computers, Television, and New Media Like Real People and Places. Cambridge: CSLI Publications, 1998, pp. 131-139.

[37] J. P. Chin, V. A. Diehl, and K. L. Norman. "Development of an instrument for measuring user satisfaction of the Human-Computer Interface," Proc. ACM Conf. Human Factors in Computing Systems (CHI 88), ACM Press, 1988. pp. 213-218, doi:ACM-O-89791-265-9/88/0004/021.

[38] S. M. Kosslyn, Graph Design for the Eye and Mind. New York: Oxford University Press, 2006. 